\title{
GUEST EDITORIAL Capturing and using design rationale
}

\author{
PAUL W.H. CHUNG ${ }^{1}$ AND RENÉ BAÑARES-ALCÁNTARA ${ }^{2}$ \\ 'Department of Chemical Engineering, Loughborough University \\ ${ }^{2}$ Department of Chemical Engineering, University of Edinburgh, Edinburgh EH9 3JL, Scotland, United Kingdom
}

\section{OVERVIEW}

Published research on design rationale (DR) goes back at least 25 years. Judging by the number of publications and the variety of application domains, there has been a significant increase in related research activities in recent years. For example, there have been other special issues on design rationale (e.g., Carrol \& Moran, 1991), workshops in international conferences (e.g., Lee, 1992; Chung \& BañaresAlcántara, 1994), and the first book on design rationale has just appeared (Carrol \& Moran, 1996).

This special issue brings together five papers discussing the representation, capture, and use of design rationale from different perspectives. A useful classification of those perspectives is proposed in Shipman and McCall (1997), where it is suggested that design rationale can be seen from the points of view of

1. Argumentation, to understand and improve the design methodologies and reasoning process of a single designer or the argumentative discourse between design participants.

2. Documentation, to maintain a decision trail for use outside the design team (in a different place or time), for example, by the regulatory authorities or the users of the artifact being designed.

3. Communication, to transfer information among members of the project team.

These perspectives are not contradictory but complementary, therefore, more than one can, and indeed should, be used in a given system.

The papers in this collection also differ in their research foci (the subarea of design rationale they address), the types of design (routine, where all the variables involved are known and the task is to find a set of acceptable values for them $v s$. innovative, where the variables in the final design are not

Reprint requests to: Dr. René Bañares-Alcántara, Department of Chemical Engineering, University of Edinburgh (KB) Edinburgh EH9 3JL, Scotland, United Kingdom. Telephone: +44(131)650-4873; Fax: +44(131)650-6551; E-mail: rene@chemeng.ed.ac.uk. known a priori) and the areas of application, which in all cases are related to engineering. Using these categorizations, the papers in this special issue can be summarized in table 1.

\section{BRIEF DESCRIPTION OF THE PAPERS IN THIS ISSUE}

Shipman and McCall (1997) present two computer systems that support the emergence of structured design arguments from design communication. While all the papers in the special issue propose the use of a specific design model, Brazier et al. (1997) presents one in more detail and shows how design rationale can be reused within such a model. King and Bañares-Alcantara (1997) propose the (semiautomatic) indexing of design objects for improved retrieval and consistency checking of design rationale structures. The focus of the two other papers is the use of design rationale: reactive documentation production (Garcia \& de Souza, 1997) and reuse of software systems (Peña-Mora \& Vadhavkar, 1997).

\subsection{PHIDIAS/HOS: Emergence of design rationale from design documentation}

The paper by Shipman and McCall (1997) provides a valuable characterization of the perspectives of design rationale in terms of their purpose and the way in which they address the issues of representation, capture, and use of design rationale. It also describes the efforts to combine the communication perspective to capture rationale (through hypertext) and the argumentation perspective to retrieve it (through taskbased indexing).

The research seeks to incorporate e-mail text, word processor documents, videos of meetings, voice mail, scanned documents, photographs, and CAD drawings to the design history representation by incremental addition of structure to the pieces of information in the system. Two systems exemplify these ideas: PHIDIAS and HOS (Hyper-Object Substrate). Incremental formalization is carried out in HOS 
Table 1

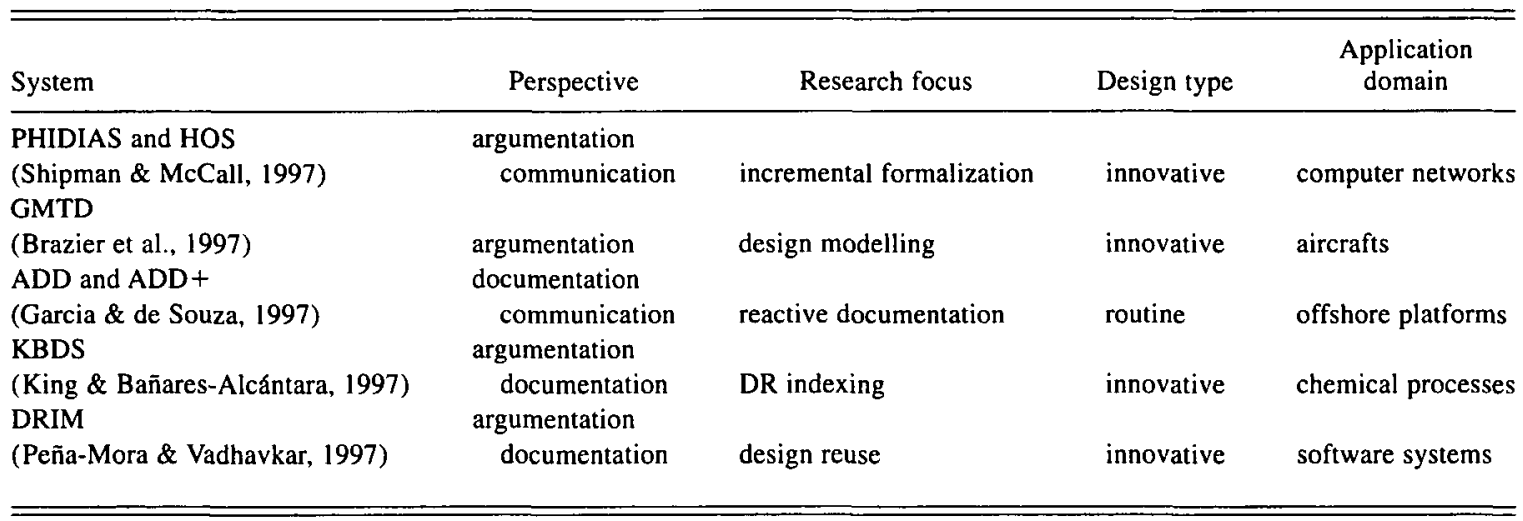

through user editable suggestions based on simple text analysis on the existing (partially) formalized knowledge. The system forms the basis for XNetwork, an environment to support computer network design.

\subsection{GMTD: Modelling design rationale}

Brazier, van Langen, and Treur (Brazier et al., 1997) propose a generic task model of design system (GMTD) for design support systems in different application domains. GTMD structures the design knowledge acquisition process into three subtasks:

- Requirement qualification set (RQS) manipulation (operating on design requirements and preferences).

- Design object description (DOD) manipulation (operating on the design object description).

- Design process coordination (determining the course of the design process).

Each subtask operates on a cycle of four steps: modification, design history update, deductive refinement, and DOD update.

GTMD is applied to the modelling of the design of a new aircraft of 60 passengers (Fokker 60 ) based on an existing design of a smaller aircraft (Fokker 50Inc) for which design rationale is available. It thus shows the reuse of design knowledge during the design of a similar artifact.

\subsection{ADD+: Active documentation of the design process}

Garcia and de Souza (1997) introduce the concept of active design documents (ADDs) as a dynamic computational representation of design rationale based on an adjustable underlying model of the artifact and its conception process. The artifact is represented by a parametric model, a common situation in routine design. ADD allows the production of reactive documentation (generated instead of being just retrieved from previous records), the generation of design decisions and rationale, and also serves as a communication medium and agent. However, ADD's interface does not organize communication into cohesive discourse and intentional message passing. For this reason, ADD has been further developed into ADD +, which generates natural language text, planned and structured using RST (rhetorical structure theory) trees and then linearized into paragraphs. ADD+ assigns intentions and beliefs (design background) to the user model from heuristic rules applied to the combination of the design dependency graph and design history.

ADD has been the basis for the development of HVAC (heating, ventilation, and air conditioning in buildings), ADDVAC (VAC in offshore platforms), and ADDPROC (VAC in offshore process plants). In turn, ADD+ is being used for the support of design of offshore oil platform process plants.

\subsection{KBDS: Extending the scope of use of design rationale records}

KBDS (King \& Bañares-Alcántara, 1997) is a support system for the conceptual (innovative) design of chemical processes. King and Bañares-Alcántara report three extensions to KBDS that address some problems derived from the variety and size of design history records, that is:

- association of a variety of complementary types of document as annotations to design objects (similar to the work described in Shipman and McCall (1997)),

- indexing of design and rationale objects with a userdefined set of keywords; this indexing is done semiautomatically by using a word-matching technique over the name, description, and textual annotations of an object and can be propagated across links, and

- consistency checking of design rationale structures based on the keywords previously assigned to their constituent nodes. 
The use and extension of KBDS is exemplified through the design of a chemical process to produce hydrofluoric acid (BUSS process) and has been used to model the design of several other process plants (hydrodealkylation of toluene, penicillin, methyl acetate), parts of a nuclear plant, and a waste water treatment plant.

\subsection{DRIM and Design Patterns: Reusing design}

Peña-Mora and Vadhavkar (1997) explore the role of design rationale in the automation of classification and retrieval of information for reuse purposes. With this objective in mind, a model for design rationale representation from the argumentation perspective previously developed (DRIM: Design recommendation and intent model) has been combined with design patterns. Design patterns are descriptions of objects and classes customized to characterize a general design problem and the core of its solution in a particular context.

The combination of DRIM and design patterns is being used in the design of reusable software systems where the knowledge reused includes software requirements, design specifications, development experience, design decisions and their rationale, computer code, and documentation.

\section{GENERAL OBSERVATIONS}

A model of the design process is important for an adequate capture of design rationale while design is carried out. However, as pointed out in Garcia and de Souza (1997), it is impossible at this point to represent the actual designer's mental model so we can, at most, aim to represent the rationalization of the process of design. The artifact model is also important because it connects the design process to the physical entity and guides, in turn, all the subsequent design decisions (Peña-Mora \& Vadhavkar, 1997).

Development of useful models of design rationale and of systems to support its representation, capture, and use benefit from the use of realistic applications (realistic in terms of the complexity of the design artifact and the size of the design development team). On the complexity side, research is focusing on bringing together diverse sources of information, and on integrating design rationale with existing design methodologies and documentation. While the trend to consider "real-world" problems is there, largescale evaluation of design rationale capture, access, and reuse is still lacking.

As could be expected, more automation is possible with routine design tasks than with innovative design ones, for example, for reactive documentation and advice (Garcia \& de Souza, 1997). Innovative design has an iterative nature resulting from an inherently limited understanding of the design issues at the conception of the design process (PeñaMora \& Vadhavkar, 1997). For this reason, it is necessary to rely on the designer's ability to navigate, relate, and understand the historical design records. We can expect the designer to perform these tasks correctly most of the time, although there is room for communication breakdowns, for example, a faulty transfer of information from the original designer to the intended user.

Finally, the papers of this issue provide examples of the important advantages that can be derived from the use of design rationale records in the tasks of:

- explanation (Brazier et al., 1997; Garcia \& de Souza, 1997; Peña-Mora \& Vadhavkar, 1997),

- indexing/navigation (King \& Bañares-Alcántara, 1997; Shipman \& McCall, 1997),

- documentation (Garcia \& de Souza, 1997; King \& Bañares-Alcántara, 1997; Peña-Mora \& Vadhavkar, 1997),

- prediction (Garcia \& de Souza, 1997),

- conflict management (Brazier et al. 1997; King \& Bañares-Alcántara, 1997; Shipman \& McCall, 1997), and

- reuse (Brazier et al., 1997; Peña-Mora \& Vadhavkar, 1997).

\section{ACKNOWLEDGMENTS}

The authors thank the authors and reviewers of the papers included in this issue.

\section{REFERENCES}

Brazier, F.M.T., van Langen, P.H.G., \& Treur, J. (1997). A compositional approach to modelling design rationale. AIEDAM $11(2), 125-139$.

Carrol, J.M., \& Moran, T.P. (1991). Introduction to the special issue on design rationale. Human-Computer Interaction 6, 197-200.

Carrol, J.M., \& Moran, T.P. (Eds.). (1996). Design rationale: Concepts, techniques and use. Lawrence Erlbaum Associates, Hillsdale, NJ.

Chung, P.W.H., \& Bañares-Alcántara, R. (Eds.). (1994). Workshop notes on Representing and Using Design Rationale. Swiss Federal Institute of Technology, Lausanne Switzerland.

Garcia, A.C.B., \& de Souza, C.S. (1997). ADD+: Including rhetorical structures in active documents. AIEDAM 1/(2), 109-124.

King, J.M.P., \& Bañares-Alcántara, R. (1997). Extending the scope and use of design rationale records. AIEDAM II(2), 155-167.

Lee, J. (Ed.). (1997). Workshop notes on Design Rationale Capture and Use, San Jose, CA.

Peña-Mora, F., \& Vadhavkar, S. (1997). Augmenting design patterns with design rationale. AIEDAM $11(2), 93-108$.

Shipman III, F.M., \& McCall, R.J. (1997). Integrating different perspectives on design rationale: Supporting the emergence of design rationale from design communication. AIEDAM $11(2), 141-154$. 